\title{
COMMENTARY
}

\section{What on earth is APRV?}

\author{
Dietrich Henzler*
}

\begin{abstract}
Partial ventilatory support modalities are ill defined and different perceptions about these modes might depend on geographic region. Exemplary on two recent publications investigating airway pressure release ventilation (APRV) in an adult ICU population, the question is investigated whether research in ventilation modes can be performed with the current definitions. The lack of precise definitions precludes drawing meaningful conclusions from these studies, as it remains unclear how these patients were actually ventilated and whether or how much spontaneous breathing was factitiously preserved. An argument is made to develop a new taxonomy of ventilation modes.
\end{abstract}

Airway pressure release ventilation (APRV) was described more than 20 years ago [1] as a mode that allows spontaneous breathing throughout the ventilation cycle. APRV is a time-cycled alternant between two levels of positive airway pressure, with the main time on the high level and a brief expiratory release to facilitate ventilation. As such, APRV may be considered a partial ventilatory support modality that has the ability to deliver the full work of breathing if needed. Although it seems that APRV is well defined, the characteristics are surprisingly unspecific, as is the way APRV is used.

Different perceptions of this mode may exist around the globe. While 'APRV' is common to users in North America, a very similar mode, biphasic positive airway pressure (BIPAP), was introduced in Europe [2]. The term APRV has also been used in American journals where, from the ventilation characteristics, BIPAP would have been the more adequate terminology [3]. To further confusion, $\mathrm{BiPAP}^{\oplus}$ (with a small ' $\mathrm{i}$ ') is a registered trademark for a noninvasive ventilation mode in a specific

\footnotetext{
*Correspondence: mail@d-henzler.de

Department of Anaesthesiology and Division of Critical Care, Queen Elisabeth II Health Sciences Centre, 10 West Victoria, 1276 South Park St, Halifax, NS, B3H 2Y9, Canada
}

ventilator (Respironics Inc.). Other names (BILEVEL, DUOPAP) have been created for legal reasons. Although similar in modality, these terms share the same shortcomings: they describe how a mode is intended to inflate the lung, rather than defining the characteristics of synchronization or the way spontaneous breathing efforts are supported.

The perceived differences between APRV and BIPAP have been described previously $[4,5]$. Essentially, APRV has a longer time phase on the high pressure level, while BIPAP usually does not exceed an inspiration:expiration time ratio of 1:1 [5]. Expiratory lung collapse is prevented in APRV by creating intrinsic positive end-expiratory pressure (PEEP) during the short expiration, while in BIPAP the PEEP is directly set with the lower pressure level. BIPAP can be applied in different ways: with unsynchronized, unsupported interfacing of spontaneous breaths ('genuine' BIPAP), with inspiratory synchronization (which is similar to a pressure-controlled, synchronized intermittent mandatory ventilation (PC-SIMV)) and with inspiratory and expiratory synchronization. Pressure support may be added on the lower level, the upper level or both.

Two recent studies have investigated the properties of these modes to improve outcome in ventilated patients in general [6] or with pulmonary contusion [7].

The first study [6] is a subanalysis from a multinational study on the change in ventilation practices [8], which reported no different outcomes for patients ventilated in APRV or assist-control (A/C). The term APRV/BIPAP was used, although the study population contained almost exclusively patients from Germany, which were likely to be ventilated in BIPAP. It remains unclear whether the authors do not perceive a difference between APRV and BIPAP from a technical or a clinical perspective.

Only the mode as set (and displayed) on the ventilator was recorded, but not whether diaphragmal movements and spontaneous breathing were actually preserved. This is crucial in interpreting the efficacy of APRV or any partial ventilatory support modality. For example, a patient ventilated in $\mathrm{A} / \mathrm{C}$ without preserved spontaneous breathing is really in volume-controlled ventilation. Concordantly, a patient in APRV/BIPAP without spontaneous breathing is ventilated in pressure-control. In a general ICU population, patients ventilated in $\mathrm{A} / \mathrm{C}$ were 
actually assisting and triggering only in 21 to 30\%, depending on the degree of oxygenation impairment [9]. This is important, for the expected benefits of APRV/ BIPAP are attributed to preserved diaphragmatic function [4]. At best, these studies compare volume versus pressure control ventilation, which showed no difference in previous studies [10]. It still may be possible that patients were breathing with partial ventilatory support, but the type and amount of such were not investigated.

Both studies reveal deep misperceptions about these modes, as $>10 \%$ of patients ventilated in APRV/BIPAP were paralyzed in one study [6], and 26\% in the other [7]. This hints at the notion that APRV/BIPAP and A/C are often used as controlled ventilation modes. Unfortunately, important ventilatory data have not been gathered or analyzed, such as triggering effort or the difference between set and actual respiratory rate.

Thus, four hypotheses are possible: 1 , APRV/BIPAP is better than A/C; 2 APRV/BIPAP is worse than A/C; 3, there is no difference between APRV/BIPAP and A/C; 4 , it is undetermined whether there is a difference between APRV/BIPAP and A/C.

As to assumption 1, Gonzalez and colleagues [6] have concluded that APRV/BIPAP reduced peak airway pressures (Ppeak). However, they compared the Ppeak of a pressure regulated mode with that of a volume regulated mode, not accounting for the added pressure from the tube resistance in volume control. Secondly, they concluded that APRV/BIPAP improved oxygenation. Although statistically significant, the clinical relevance is uncertain. The effects of APRV/BIPAP on oxygenation are well described, in APRV by increasing mean airway pressure (as function of prolonged inspiratory time) [4] and in 'genuine' BIPAP by increasing transpulmonary pressure [11]. No difference was noted in clinical outcomes for the whole study population, but a more relevant cohort of patients with severe oxygenation impairment has not been analyzed separately.

On the contrary, Walkey and colleagues [7] have found that in North American patients the rate of ventilatorassociated pneumonia was reduced with APRV, compared to with 'conventional ventilation', mostly volume $\mathrm{A} / \mathrm{C}$. It is surprising that no other factors known to be predictors of ventilator-associated pneumonia (that is, days on ventilator, lung protective ventilation settings) were of significance.

The same considerations apply to assumption 2, which has to be rejected as well.

Geographical considerations might be applicable to evaluate assumption 3. The majority of the analyzed patients from [6] were most likely ventilated in BIPAP with a normal inspiratory:expiratory ratio. The different ways BIPAP can be applied were not recorded, although these choices might have important implications. For example, genuine BIPAP has the greatest effect on gas exchange, but dramatically increases the work of breathing compared to $\mathrm{A} / \mathrm{C}[12,13]$. Walkey and colleagues [7] do not provide more precise data, as only the inspiratory:expiratory ratio was reported for the APRV specifications. At least two different APRV modes have been used in this study ('BILEVEL' (Covidien) and 'APRV' (Draeger)), which can be set differently in their triggering, expiratory synchronization or support of spontaneous breaths characteristics. Thus, inhomogeneous groups were compared and assumption 3 cannot be supported.

What remains is assumption 4 . What the clinician would have liked to know is how these modes were used, when and why in the course of treatment they were applied, when and why patients were switched to another mode, or how much of the spontaneous breathing was allowed. In many institutions that had contributed to the study [6], patients were typically started on BIPAP early in the course of treatment without or with very little spontaneous breathing, and were gradually allowed to increase spontaneous breathing. However, it is unclear whether this approach is better than a more traditional one, and this question cannot be answered if the confusions about the true ventilation modalities persist.

In conclusion, the calamity of ventilation research is revealed in a way such that inhomogeneous groups of full or partial ventilatory support modalities cannot be compared well. Could the notion that APRV is effective in improving outcome in North America, but not in Europe, be explained by a different way that APRV is applied? What becomes clear though is that more precise definitions distinct from the traditional taxonomy of ventilation modalities are desperately needed for research and clinical application.

\section{Abbreviations}

$A / C$, assist control ventilation; APRV, airway pressure release ventilation; BIPAP, biphasic positive airway pressure; PEEP, positive end-expiratory pressure; Ppeak, peak inspiratory airway pressure.

\section{Competing interests}

The author declares that they have no competing interests.

\section{Published: 31 January 2011}

\section{References}

1. Downs JB, Stock MC: Airway pressure release ventilation: a new concept in ventilatory support. Crit Care Med 1987, 15:459-461.

2. Baum M, Benzer H, Putensen C, Koller W, Putz G: [Biphasic positive airway pressure (BIPAP)-a new form of augmented ventilation]. Anaesthesist 1989, 38:452-458.

3. Putensen C, Zech S, Wrigge H, Zinserling J, Stuber F, von Spiegel T, Mutz N: Long-term effects of spontaneous breathing during ventilatory support in patients with acute lung injury. Am J Respir Crit Care Med 2001, 164:43-49.

4. Putensen $\mathrm{C}$, Wrigge $\mathrm{H}$ : Clinical review: Biphasic positive airway pressure and airway pressure release ventilation. Crit Care 2004, 8:492-497.

5. Rose L, Hawkins M: Airway pressure release ventilation and biphasic positive airway pressure: a systematic review of definitional criteria. Intensive Care Med 2008, 34:1766-1773.

6. Gonzalez M, Arroliga AC, Frutos-Vivar F, Raymondos K, Esteban A, Putensen C, 
Apezteguia C, Hurtado J, Desmery P, Tomicic V, Elizalde J, Abroug F, Arabi Y, Moreno R, Anzueto A, Ferguson ND: Airway pressure release ventilation versus assist-control ventilation: a comparative propensity score and international cohort study. Intensive Care Med 2010, 36:817-827.

7. Walkey AJ, Nair S, Papadopoulos S, Agarwal S, Reardon CC: Use of airway pressure release ventilation is associated with a reduced incidence of ventilator-associated pneumonia in patients with pulmonary contusion. J Trauma 2010 [Epub ahead of print].

8. Esteban A, Ferguson ND, Meade MO, Frutos-Vivar F, Apezteguia C, Brochard L, Raymondos K, Nin N, Hurtado J, Tomicic V, Gonzalez M, Elizalde J, Nightingale P, Abroug F, Pelosi P, Arabi Y, Moreno R, Jibaja M, D’Empaire G, Sandi F, Matamis D, Montanez AM, Anzueto A: Evolution of mechanical ventilation in response to clinical research. Am J Respir Crit Care Med 2008, 177:170-177.

9. Henzler D, MacDonald M, Gilles A, Julien L, McMullen S: Mechanical ventilation parameters associated with assisted breathing in intubated adult intensive care patients. Am J Respir Crit Care Med 2010, 181(Suppl):A3002

10. Esteban A, Alia I, Gordo F, de Pablo R, Suarez J, Gonzalez G, Blanco J: Prospective randomized trial comparing pressure-controlled ventilation and volume-controlled ventilation in ARDS. For the Spanish Lung Failure Collaborative Group. Chest 2000, 117:1690-1696.

11. Henzler D, Dembinski R, Bensberg R, Hochhausen N, Rossaint R, Kuhlen R: Ventilation with biphasic positive airway pressure in experimental lung injury. Influence of transpulmonary pressure on gas exchange and haemodynamics. Intensive Care Med 2004, 30:935-943.

12. Henzler D, Pelosi P, Bensberg R, Dembinski R, Quintel M, Pielen V, Rossaint R, Kuhlen R: Effects of partial ventilatory support modalities on respiratory function in severe hypoxemic lung injury. Crit Care Med 2006, 34:1738-1745

13. Calzia E, Lindner KH, Witt S, Schirmer U, Lange H, Stenz R, Georgieff M: Pressure-time product and work of breathing during biphasic continuous positive airway pressure and assisted spontaneous breathing. Am J Respir Crit Care Med 1994, 150:904-910.

doi:10.1186/cc9419

Cite this article as: Henzler D: What on earth is APRV? Critical Care 2011 15:115 